\title{
Shape-based Scenario Generation using Copulas
}

\author{
Michal Kaut* \\ michal.kaut@himolde.no
}

\author{
Stein W. Wallace* \\ stein.w.wallace@himolde.no
}

13 September 2006

\begin{abstract}
The purpose of this article is to show how the multivariate structure (the "shape" of the distribution) can be separated from the marginal distributions when generating scenarios. To do this we use the copula. As a result, we can define combined approaches that capture shape with one method and handle margins with another. In some cases the combined approach is exact, in other cases, the result is an approximation. This new approach is particularly useful if the shape is somewhat peculiar, and substantially different from the standard normal elliptic shape. But it can also be used to obtain the shape of the normal but with margins from different distribution families, or normal margins with for example tail dependence in the multivariate structure. We provide an example from portfolio management.
\end{abstract}

Keywords: stochastic programming, scenario-generation, copula, shape, multivariate structure.

\section{Introduction}

Stochastic programming has become a common tool to study and model decision problems with the presence of uncertainty. These models are usually based on the use of multivariate probability distributions describing the uncertainty in the input data. The exact or approximating methods that are important for applications mainly deal with discrete empirical probability distributions that are described by a list of realizations (called scenarios) and related probabilities. See Wallace and Ziemba (2005) for a discussion of modeling as well as applications.

In most applications, the multivariate distributions do not come in a form suitable for the optimization model, being either continuous, discrete with too many data points, or specified by a set of statistical properties. Hence, to use a stochastic programming model, one has to transform the given distribution to scenarios-a process known as scenario generation. There exist many different scenario-generation methods, each with its strengts and weeknesses, see for example Dupačová et al. (2003), Høyland and Wallace (2001), Høyland et al. (2003), Pflug (2001), Römisch and Heitsch (2003), and Heitsch and Römisch (2005). For an overview, see Dupačová et al. (2000).

In recent years, we have been studying - and using-scenario-generation methods that use the first four moments to describe the marginal distributions and the correlation matrix to describe the multivariate structure — see Høyland et al. (2003), Kaut et al. (2003). While our experience shows that in many applications four moments provide a sufficient control over the marginal distributions, the usefulness of correlations is much more limited. The reason is that a correlation-or more precisely the Pearson's correlation coefficient—describes only the degree of linear dependence between two

${ }^{*}$ Molde University College, P.O. Box 2110, N-6402 Molde, Norway 
random variables. It does not capture any non-linear dependencies, and it does not tell us anything about the "shape" of the bivariate structure. In fact, using the Pearson correlation often implicitly means assuming the elliptical shape of the normal distribution.

On the other hand, several recent studies-e.g. Hu (2006), Longin and Solnik (2001), Patton $(2002,2004)$ - point out that some financial data are not elliptical, showing for example higher correlations for downturns than for upturns (all markets tend to crash together). This is illustrated in Figure 1, which shows a scatterplot of daily returns of US and UK small cap stocks, using data from $\mathrm{MSCI}^{1}$. To demonstrate that the asymmetry does not come from the marginal distributions, we present also a plot of returns with margins transformed to the standard normal distribution.
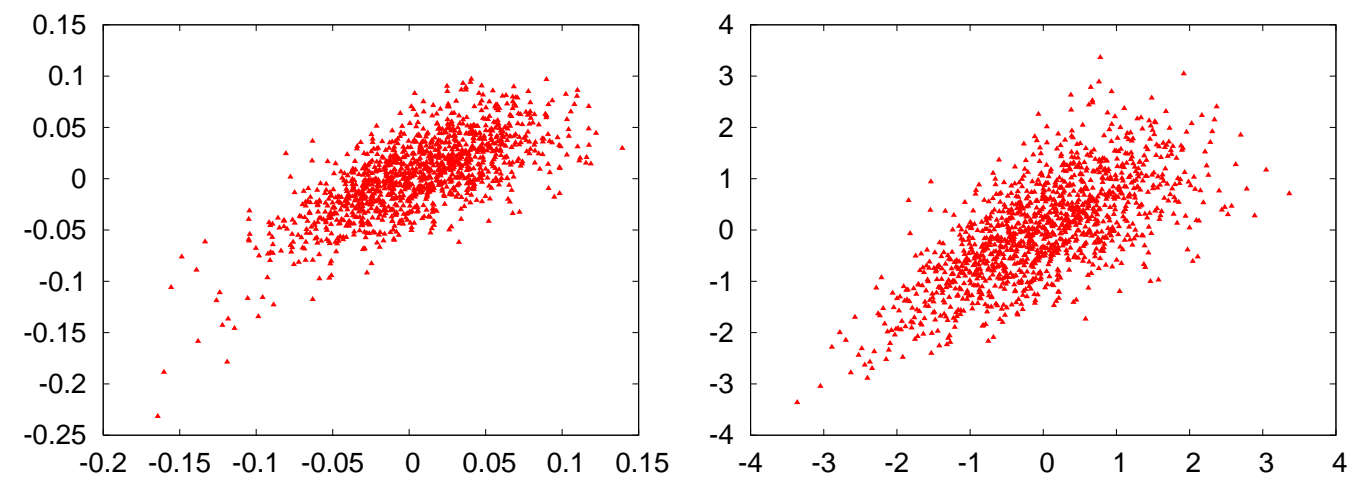

Figure 1: Scatter plot of daily returns of US small caps vs. UK small caps. The left figure shows the actual data, the right figure the data with margins transformed to standard normal distribution, to demonstrate that the asymmetry is not caused by the marginal distributions.

While we are not aware of studies from other areas, we find it likely that structures significantly different from those of the normal distribution can be found in many practical settings.

In this paper, we propose a general framework that can, at least theoretically, generate scenarios with any multivariate structure. In addition, we propose several methods that fit into the framework and can be used in different cases.

The framework is based on copulas, a concept that has been used in statistics and finance for some time — see for example Bouyé et al. (2000), Clemen and Reilly (1999), Rosenberg (2003)—yet remains virtually unknown in the rest of the OR community. To our knowledge, copulas have not yet been presented as a basis for scenario generation.

The rest of the paper is organised as follows: In the first section, we present the main results from the copula theory and discuss what it can offer for the scenario-generation problem. In the next section, we present the general framework, with more details coming in Section 3. The framework is then exemplified in Section 4.

\section{Copulas and their place in scenario generation}

This section first presents the notion of a copula, then presents the main results from copula theory, and finally shows what this means for scenario generation.

\footnotetext{
${ }^{1}$ Morgan Stanley Capital International Inc.; www.msci.com/equity/.
} 


\subsection{Definitions and main results}

The name copula was first used in Sklar (1959) to describe "a function that links a multidimensional distribution to its one-dimensional margins". The mathematical formulation comes from Sklar (1996) and Nelsen (1998).

An $n$-dimensional copula is the joint cummulative distribution function (CDF) of any $n$-dimensional random vector with standard uniform marginal distributions, i.e. a function $C:[0,1]^{n} \rightarrow[0,1]$. Sklar's theorem states that for any $n$-dimensional CDF $F$ with marginal distribution functions $F_{1}, \ldots, F_{n}$, there exist a copula $C$ such that

$$
F\left(x_{1}, \ldots, x_{n}\right)=C\left(F_{1}\left(x_{1}\right), \ldots, F_{n}\left(x_{n}\right)\right) .
$$

Moreover, if all the marginal CDFs $F_{i}$ are continuous, then $C$ is unique. For the proof, see Sklar (1996).

An immediate consequence of the theorem is that, for every $\boldsymbol{u}=\left(u_{1}, \ldots, u_{n}\right) \in[0,1]^{n}$,

$$
C\left(u_{1}, \ldots, u_{n}\right)=F\left(F_{1}^{-1}\left(u_{1}\right), \ldots, F_{n}^{-1}\left(u_{n}\right)\right),
$$

where $F_{i}^{-1}$ is the generalised inverse of $F_{i}$.

An important property of the copula is that it does not change under strictly increasing transformations of the margins. This allows us to transform margins from one continuous distribution to another, without changing the copula: if the margin $\tilde{X}_{i}$ has a CDF $F_{i}$, then $G_{i}^{-1}\left(F_{i}\left(\tilde{X}_{i}\right)\right)$ has $\operatorname{CDF} G$, and the copula does not change since both $F_{i}$ and $G_{i}^{-1}$ are increasing.

This also means that any statistical property that depends only on the copula is invariant to strictly increasing transformations of the margins. An example of such a statistics is the Spearman's (rank) correlation-while the 'standard' Pearson's linear correlation is invariant only under positive linear transformations.

For the simplest example of a copula, consider two independent random variables $\tilde{X}_{1}$ and $\tilde{X}_{2}$ with $F\left(x_{1}, x_{2}\right)=F_{1}\left(x_{1}\right) F_{2}\left(x_{2}\right)$. The associated copula is $C\left(u_{1}, u_{2}\right)=u_{1} u_{2}$, i.e. the CDF of two independent standard uniform random variables.

Another example is the Gaussian copula, i.e. the copula of an $n$-variate standard normal distribution with correlation matrix $\Sigma$ :

$$
C_{\Sigma}\left(u_{1}, \ldots, u_{n}\right)=\Phi_{\Sigma}\left(\Phi^{-1}\left(u_{1}\right), \ldots, \Phi^{-1}\left(u_{n}\right)\right),
$$

where $\Phi_{\Sigma}$ is the joint CDF of the multivariate normal distribution.

For more information about copulas, see for example Clemen and Reilly (1999), Nelsen (1998), Sklar $(1959,1996)$. In addition, substantial information can be found in the help file of Matlab ${ }^{\circledR} 7$, in the section "Simulating Dependent Random Variables Using Copulas".

\subsection{Advantages of using copulas for scenario generation}

Since the copula is obtained from the joint CDF by transforming the margins to the standard uniform distribution, it can be seen as the joint distribution stripped of all the information about the margins. What is left is information about the multivariate structure-none of this information is lost by transforming the margins.

Copulas therefore allow us to de-couple the margins from the overall multivariate structure, and model these two independently: we start by modelling/generating the copula, i.e. the multivariate 
structure with uniform margins. This can be done, for example, by generating or sampling a distribution with the desired structure without regard to the marginal distributions involved. Thereafter we transform the margins to the standard uniform distribution to obtain the copula. Once we have the copula, we transform the margins again to get the desired marginal distributions. This opens some new possibilities for scenario generation, some of which are listed here:

\section{Combining different (standard) copulas and margins}

If we compare the normal distribution with $t$ distributions (with sufficiently small number of d.o.f.), the most obvious difference is in the tails of the marginal distributions. There is, however, also one important difference between the two implied copulas, i.e. between the multivariate structures: the $t$ distribution exhibits a tail dependence, defined as follows: A bivariate random vector $\left(\tilde{X}_{1}, \tilde{X}_{2}\right)$ is lower-tail dependent if its lower-tail dependendence coefficent

$$
\lambda_{L}=\lim _{v \rightarrow 0^{+}} \mathrm{P}\left\{\tilde{X}_{1} \leq F_{\tilde{X}_{1}}^{-1}(v) \mid \tilde{X}_{2} \leq F_{\tilde{X}_{2}}^{-1}(v)\right\}
$$

is strictly positive. Upper-tail dependence is defined analogously. The normal distribution is tailindependent as long as the correlation is stricly smaller than one. This means that the extreme events are always independent, so we won't get really extreme scenarios where everything goes awry.

However, since the tail dependence is a function of the copula and does not depend on the marginal distributions, it is possible to create, for example, distributions with normal margins and $t$ copula structure, i.e. normal margins with tail dependence. Note that the margins are not limited to normal distribution, each margin can even have a different type of distribution.

\section{Introducing asymmetry}

Instead of the standard $t$ copula, we can use a copula from one of the skew- $t$ distributions. These distributions allow for several types of asymmetric dependencies, the most important of which is the possibility of having higher correlation on the down-turn than on the up-turn-an effect observed, for instance, in some financial data.

Unfortunately, there are several different skewed versions of $t$ distributions, each with different strenghts and weaknesses. For information about the most important ones, see for example Adcock (2003), Azzalini and Capitanio (2003), Bauwens and Laurent (2002), Demarta and McNeil (2005), Jondeau and Rockinger (2000), Jones (2001). In addition, there is the noncentral $t$ distribution and Pearson Type IV distribution. For information on the latter, see Heinrich (2004).

Assuming that we are able to estimate the parameters for the chosen skew- $t$ distribution, we can generate a sample from this distribution and then transform the margins, obtaining asymmetric dependency with arbitrary marginal distributions.

\section{Using principal components}

In many applications, it can be argued that there are too many random variables in the model, and the dimension could (and should) be reduced by techniques like principal components analysis (PCA). In addition to decreasing the dimension of the stochastic vector, the principal components are also uncorrelated-and therefore, in the case of normal distributions, independent. This means that scenarios for the individual principle components can be generated independently, converting the multivariate scenario generation to a much easier univariate generation problem. (The univariate margins can be combined into the multivaraite vector in an all-against-all fashion, or by a random coupling of 
the margins. With the former, the number of scenarios grows exponentially with the dimension of the random vector, often resulting in a need to use a scenario-reduction procedure afterwards, while the latter yields scenarios that are only approximately indepent.)

For other than normal distributions, however, the principal components are only uncorrelated, so there is still some dependence structure to be captured. As an example, see Figure 2, where the two principle components are clearly not independent, despite having zero correlations. Yet, as long as we use correlations as the only description of the multivariate structure, we are not able to make the distinction between uncorrelated and independent random variables and therefore can not model the structure properly.

It is therefore easy to forget the distinction between uncorrelatted an independent. So much so, that it is possible to find papers that either claim that principal components are independent - see, for example, DeMiguel and Mishra (2006), - or generate them as independent without explicitly saying so-as in Jamshidian and Zhu (1996). In addition, even when authors are aware of the problem they might still assume normality and treat the principal components as independent, most likely for the lack of better tools: Frauendorfer and Schürle (2005), Halldin (2002), Topaloglou et al. (2002).
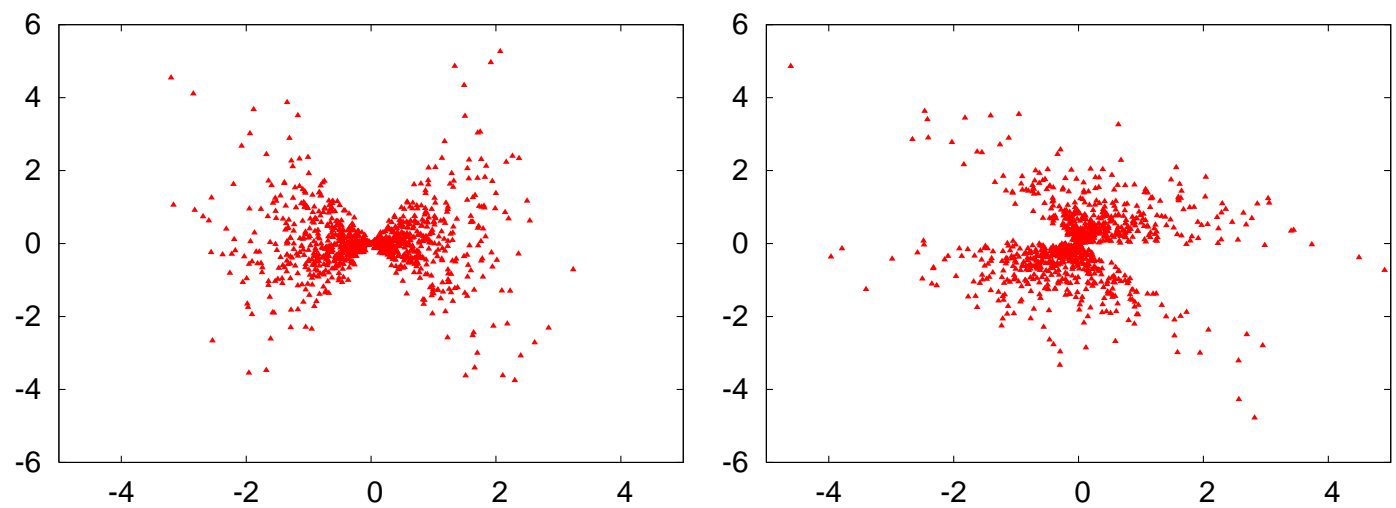

Figure 2: Bi-variate distribution with margins $\tilde{x}_{1}=\tilde{\xi}_{1}, \tilde{x}_{2}=\tilde{\xi}_{1} \tilde{\xi}_{2}$, with $\tilde{\xi}_{1}, \tilde{\xi}_{2} \sim \mathrm{N}(0,1)$, independent. The left figure shows a sample from the random vector $\tilde{\boldsymbol{x}}=\left(\tilde{x}_{1}, \tilde{x}_{2}\right)$, the right figure its principal components, scaled to variance equal to one. The principal components were computed from a sample of 25,000 points, but the plots show only the first 1000 points for better readability.

Copulas, on the other hand, are capable of capturing the structure properly, allowing thus the use of principal components also for non-normal distributions. It is also possible that the distributions of principal components have qualitatively different structures than those of the underlying random variables, something that could be taken care of by the copula-based approach. This is, however, out of the scope of this paper and is left for future research.

\subsection{Stability and Optimality gap}

The ultimate test of the quality of a scenario tree will be how well it fits the corresponding stochastic program. It is not the purpose of this paper to discuss that issue, but we would like to point out that there are several ways to think about quality of a scenario tree. Obvious possibilities are to compare the scenario tree directly to the underlying distribution, using metrics from probability theory-see for example Heitsch et al. (2006), - or comparing (optimal) values of the relevant optimization problem, hence using the optimization problem as a metric. In the latter case, the performance of the scenariobased solutions can be evaluated using either a simulator—as in Kaut and Wallace (2003), — or a 
confidence interval obtained by solving several optimization problems, see Bayraksan and Morton (2005), Chiralaksanakul and Morton (2004), Linderoth et al. (2002).

\section{The algorithm}

In this section, we present the algorithm for scenario-generation, i.e. for generating a discrete multivariate sample satisfying given properties/characteristics. In particular, we focus on the case when the distribution is given in the form of historical data — but the algorithm can be, possibly with some adjustments, used in other cases as well.

\subsection{Basic structure}

Assume that we start with historical data set $\mathbf{D} \in \mathbb{R}^{n, n_{D}}$, where $n$ is the number of random variables and $n_{D}$ is the length of the data set. We want to "replicate" $\mathbf{D}$ by $n_{S}$ scenarios. i.e. by a discrete $n$-dimensional random vector $\tilde{\boldsymbol{X}}$ with $n_{S}$ outcomes per variables. Typically, $n_{S}<n_{D}$ or even $n_{S} \ll n_{D}$. The algorithm/framework is then as follows:

1. Transform the data set $\mathbf{D}$ to a new set $\mathbf{C}$ with standard uniform margins by

$$
c_{i j}=F_{i}\left(d_{i j}\right), \quad i=1 \ldots n, j=1 \ldots n_{D},
$$

where $F_{i}$ is an estimate of the (univariate) distribution function of the $i$-th random variable. In our case, we use empirical CDFs from the data set, so columns of $\mathbf{C}$ consists of the ranks of the columns of $\mathbf{D}$, scaled to the interval $(0,1)$ :

$$
c_{i j}=\frac{1}{n_{D}}\left(\operatorname{rank}\left(u_{i j}, \boldsymbol{u}_{i}\right)-0.5\right), i=1, \ldots, n,
$$

where $\operatorname{rank}\left(x_{s}, \boldsymbol{x}\right)$ is the rank (order) of value $x_{s}$ in a vector $\boldsymbol{x}$, with

$$
1=\operatorname{rank}(\min (\boldsymbol{x}), \boldsymbol{x}) \leq \operatorname{rank}\left(x_{s}, \boldsymbol{x}\right) \leq \operatorname{rank}(\max (\boldsymbol{x}), \boldsymbol{x})=n_{D} .
$$

In other words, $\mathbf{C}=\left(c_{i j}\right)$ is a copula corresponding to the data set $\mathbf{D}$. We will refer to it as the "historical copula".

2. Based on the historical copula $\mathbf{C}$, create the copula for the scenarios, i.e. a sample $\boldsymbol{U}$ from an $n$-variate discrete random vector $\tilde{U}$ with standard uniform margins and structure close to the one of $\mathbf{C}$. This can be done in several different ways, details will be discussed in Section 3:

- Sampling from the historical copula $\mathbf{C}$.

- Using some parametric family of copulas, with parameters estimated from the historical copula.

- Creating the structure by coupling of the ranks.

3. Once we have the "scenario copula" $\boldsymbol{U}$, we have to transform the margins from the uniform to the desired distribution. The options are:

- Using the empirical CDF from the historical data.

- Using some parametric family of distributions, with parameters estimated from the historical data. The margins are then obtained by the inversion method. 
- Compute the moments from the historical data and use some moment-matching method to transform the scenarios to match the moments: For example, to match the first four moments we use the cubic transformation from Fleishman (1978), in the way described in Høyland et al. (2003).

\subsection{Details and comments}

\section{Controlling the correlations/covariances}

While the change of the margins does not change any copula-based measure (like Spearman's rank correlations), it will change the Pearson's correlations. The difference is generally hard to predict as it depends on several factors.

For example, if we sample the copula from historical data and then transform the margins to the distributions equal to (or close to) the historical ones, the difference can be expected to be small-in fact, it will converge to zero as the sample size increases (provided we have enough data).

On the other hand, if we obtain the copula from one multivariate distribution (like $t$-distribution) and then transform the margins to another distribution (like normal), there will always be a difference in the correlations of the starting distribution and the final scenarios. The size of the difference will depend on the difference (in shape) between the initial and the final marginal distributions.

If we need exact correlations, we can use the moment-matching algorithm from Høyland et al. (2003) as a post-process, setting the correlations to the desired values, while preserving (most of the) shape of the margins by controlling their first four moments. Since the process involves Cholesky transformation of the data, it will invariably distort the copula. The severity of the distortion will depend on the size of the errors to be corrected, small corrections should not change the structure noticeably.

\section{Fixing margins of the copula sample}

If we use sampling to get the copula for the scenarios in Step 2 of the algorithm, we face the usual pitfalls of the sampling approach: even if the procedure is unbiased in the limit, for small $n_{S}$ it can be very unstable. Fortunately, in the case of copulas the margins follow the standard uniform distribution and can be adjusted accordingly.

In our case, we have stretched each margin so that the points fell into the centers of subintervals of length $1 / n_{s}$ :

$$
u_{i s}^{\prime}=\frac{1}{n_{S}}\left(\operatorname{rank}\left(u_{i s}, \boldsymbol{u}_{i}\right)-0.5\right) .
$$

Since the stretching constitutes a monotonous transformation of the margins, it does not change the copula.

A word of caution: while fixing the margins improves the stability, it also causes the margins to have the same values in all scenario trees (assuming the transformation in Step 3 is deterministic). The only difference is how the margins are connected to form the multivariate distribution-the copula. If this, for any reason, causes a problem to the modeller, but the sampled margins $\boldsymbol{u}_{i}$ are too unstable to be used unchanged, it is possible to use weighted averages $\alpha \boldsymbol{u}_{i}^{\prime}+(1-\alpha) \boldsymbol{u}_{i}$ instead, with a suitable value for $\alpha$. 
1 Using moment-matching for the transformation of margins

3

If we use a cubic transformation to transform the margins to a distribution with specified first four moments, it is important to realise that the cubic transformation is not necessarily strictly increasing, so it can change the copula. In addition, the transformation may not even be possible in one step, since the 'distance' of the target distribution from the uniform distribution may be too big.

To minimise this danger, we should first transform the margins to a distribution that is closer to the target, using an (increasing) inverse CDF that does not change the copula. As a result, the cubic transformation will be closer to identity, decreasing the possible distortion of the copula. Already transforming from uniform to normal will help for most of the common distributions, but we could do even better with some four-parameter distributions like skew- $t$, non-central $t$, or Pearson Type IV—-see Section 1.2 for a list of related papers.

\section{Relation to the moment-matching algorithm by Høyland et al.}

If we require control of moments and correlations, we can use the scenarios obtained by the copulabased method as a starting point for the moment-matching algorithm from Høyland et al. (2003). In this context, the algorithm can be seen as a series of transformations to transform a starting sample to a sample with distribution with specified first four moments and a correlation matrix. Two transformations are used to do this: a cubic transformation to correct the moments of the margins and a matrix transformation using a Cholesky component of the correlation matrix to correct the correlations.

Compared to using the moment-matching algorithm only, using the copula-based approach to get a starting point should improve the algorithm in the following ways:

- In the current implementation of the algorithm, we start with discretized normal variates that are paired randomly and then transformed to the specified correlation matrix. Hence, there is no control over the initial structure and even a simple sampling from the historical copula should give better results.

- Just as the matrix transformation distorts the margins, the cubic transformation distorts the copula structure, with size of the distortion increasing with the distance of the starting and the target distributions. Therefore, even starting with approximate marginal distributions from the copula-based method should be better than starting with the normal margins (obviously unless the target distribution is normal).

Both these improvements should lead to better stability and/or better solutions of the optimization model, for a given number of scenarios. The extent (and indeed the presence) of the improvement will be tested in Section 4.

\section{Methods for constructing the scenario copula}

In this section, we present the methods used for the generation of the scenario copula, i.e. the scenario distribution with uniform margins. For each method, we discuss where it could be used and present a numerical example.

\subsection{Sampling}

The easiest option is to sample the values of $\tilde{\boldsymbol{U}}$ from the historical copula $\mathbf{C}$. It is also the only method that guaratees the correct distribution in the limit (in this case, as $n_{S}$ approaches $n_{D}$ ). Note that we 
sample only the copula (the multivariate structure), as the marginal distributions are fixed later in Step 3 of the algorithm. This is a major difference from the standard sampling method, where we do not have any control over the marginal distributions (except, perhaps, for simple corrections of means and variances).

\subsection{Using a standard copula}

Another option is using some standard copula: copulas, just like distributions, have many parametric families with specialized methods for generation. Once we have decided for a particular copula, we have to estimate its parameters from the historical copula $\mathbf{C}$ and then use an appropriate method to create a sample from the copula. The best source of information on copula families is probably Nelsen (1998), other options include Bouyé et al. (2000), Hu (2006), Romano (2002).

In addition to the copula families, it is possible to use copulas from some standard distribution like normal or $t$, or the skewed versions of $t$ distributions mentioned in Section 1.2. In this case, we generate a sample from the given distribution and then transform it to a copula in the same way as we did with the data in Step 1 of the algorithm in Section 2.

Note that the transformation to copula removes all information of the marginal distributions, so only the copula (structure) of the chosen distribution remains. This means, for example, that we do not have to estimate the scale parameters, as they do not influence the copula. In other words, the normal copula depends only on the correlations, the $t$ copula in addition on the degrees of freedom, and the skewed version of $t$ in addition on the skewness parameter(s). Furthermore, the skewness parameter(s) are used only to control the assymetry of the skewed- $t$ copulas, they have no relation to the skewness of the final distribution (again, because the marginal distributions are removed by the transformation to copula). This is illustrated in Figure 3 where the distribution remains skewed even when the margins are transformed to the standard normal distributions. For comparison, we present also a distribution obtained by combining the skewed- $t$ margins with a standard normal copula. Note that unlike the skewed- $t$ distributions, the extreme values do not happen together when we use the same margins with the normal copula. This is in concordance with the fact that the normal copula does not exhibit tail dependance, as mentioned in Section 1.2. For more information on using copulas of standard distributions, see for example Demarta and McNeil (2005), Romano (2002).

\subsection{Coupling of the ranks of the margins}

Any finite discrete copula can be seen as a set of couplings of the ranks of the margins: the first element could, for example, consist of the second smallest value of the first margin and the $13^{\text {th }}$ smallest value of the second margin, etc. Hence, to generate a copula it is enough to generate a set of couplings with given properties. This would lead to a "property-matching-type" of algorithm for the copula. Development of such an algorithm is, however, out of the scope of this paper as is left for future research.

\section{Case study - portofilo optimization with CVaR constraint}

In this section, we test several variants of the scenario-generation method on a portfolio optimization model with a CVaR constraint. It is a one-period LP model, with positive variables (positions) that sum up to one. The LP formulation of the CVaR constraint comes from Rockafellar and Uryasev (2000) and Uryasev (2000). 
original distribution
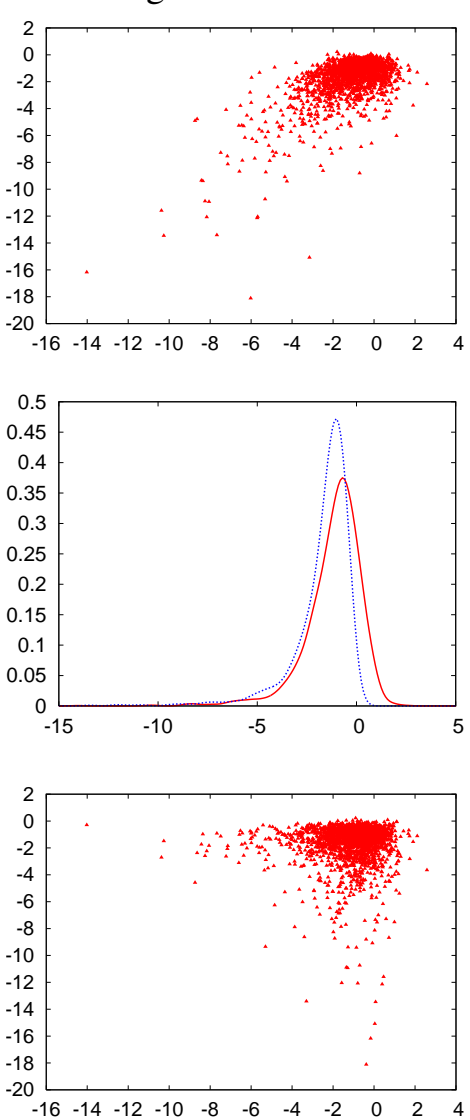

copula
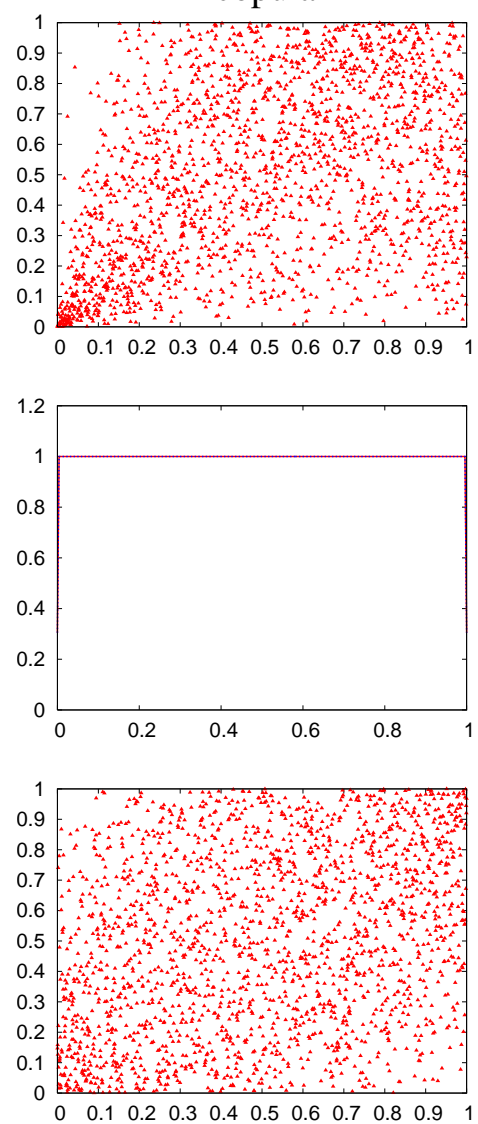

normal margins
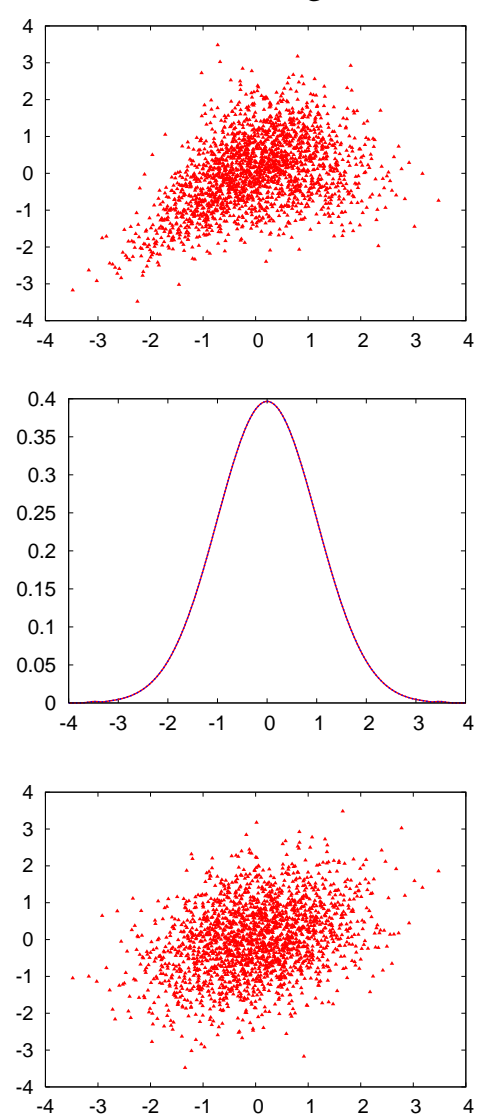

Figure 3: Skewed- $t$ distribution and copula, using a skewed- $t$ variant from Azzalini and Capitanio (2003) with 5 degrees of freedom and skewness parameters $(-0.5,-0.9)$. The first two rows show the two-dimensional scatter plots and marginal densities, respectively. The third row shows a distribution obtained by combining the skewed- $t$ margins with a standard normal copula. In other words, the marginal distribution in the second row correspond both to the first and the third row. Note that the bottom-right figure is a standard normal distribution. The reason there seems to be only one line in the second and third figure in the second row is that in those cases both margins have the same distributions, $\mathrm{U}(0,1)$ and $\mathrm{N}(0,1)$, respectively.

The CVaR model has been chosen because it can be expected to react to differences in the shape of the distribution, particularly the shape of lower tail of the return distribution. Two sets of data were used for the model: the main data set consists of daily prices of seven stock indices and three government bonds, from 1987-07-09 to 2005-04-05 (4476 points). This data set was kindly provided by Kjetil Høyland from DNB Nor, Oslo, Norway. The second data set consists of 1302 daily prices of 10 stock indices, obtained from MSCI.

We have tested several version of the copula-based approach, as well as sampling and the momentbased approach from Høyland et al. (2003). In addition, several post-processing (adjusting) methods were tried to improve the scenarios.

All the copula-based approaches used sampling in Step 2 of the algorithm presented in Section 2, and differed in the implementation of the transformation of the margins in Step 3 of the algorithm: 
one option was to transform the margins using the inverse of the empirical CDFs from the data. ${ }^{2}$ Alternatively, we can transform margins to some standard distribution with known CDF (in our case normal) and then use a post-process (in our case moment-matching) to get the correct margins. In addition, we tested using the fixed margins, as described in Section 2.2.

This gives the following scenario-generation methods:

histRet Sample from historical returns.

mom+cor Use the moment-matching alg. from Høyland et al. (2003) to match the first four moments of the margins plus the correlation matrix.

histCopNorm Sample from copula and transform margins to $\mathrm{N}(0,1)$.

histCopFixNorm The same, using fixed margins of the copula.

histCopICdf Sample from copula and transform margins using the inverse of the historical CDF.

histCopFixICdf The same, using fixed margins of the copula.

Note however that 'histCopICdf' is the same as 'histRet': first we get the sample from the historical returns and apply the empirical CDF to transform it to copula. Then we apply the inverse of the empirical CDF to get the correct distribution of margins, leaving us with the original sample. This method will therefore not be considered in the tests. Note also that 'histCopFixICdf' is different, as we change the copula sample before using the inverse $\mathrm{CDF}$.

An obvious choice of a post-process is to adjust the margins to correct their means and variancesthis can be done by a simple linear transformation. Alternatively, we can use the moment-matching method from Høyland et al. (2003) to correct the moments of the margins and the correlation matrix. ${ }^{3}$

This gives the following post-processing methods:

\section{none}

meanVar Correct means and variances of the margins.

moments Use the moment-matching method to correct the first four moments of the margins.

mon+cor Correct the correlation matrix, in addition to the moments.

Not all combinations of scenario-generation and post-process methods are possible and sensible, 27 so only the following 13 methods were tested:

\begin{tabular}{rccl} 
& initialization & post-process & comment \\
\hline 0 & histRet & none & standard sampling \\
1 & histRet & meanVar & sampling with correction of means and variances \\
2 & histRet & moments & \\
3 & histRet & mom+cor & \\
4 & mom+cor & none & 'pure' moment-matching, as done in Høyland et al. (2003) \\
5 & histCopFixNorm & moments & \\
6 & histCopFixNorm & mom+cor & \\
7 & histCopFixICdf & moments & \\
8 & histCopFixICdf & mom+cor & \\
9 & histCopFixICdf & none & sampling from the copula, using fixed uniform margins \\
10 & histCopFixICdf & meanVar & \\
11 & histCopNorm & moments & \\
12 & histCopNorm & mom+cor & \\
\end{tabular}

\footnotetext{
${ }^{2}$ In the actual implementation we have interpolated the inverse empirical CDF using cubic splines to get values at points different from the data points.

${ }^{3}$ The algorithm allows specifying a starting point (distribution) for the iteration process.
} 


\footnotetext{
4 The GNU Linear Programming Kit, see http://www.gnu.org/software/glpk/glpk.html. GNU MathProg is an implementation of a subset of AMPL. Precompiled version of GLPK for Windows can be obtained from http:// gnuwin32. sourceforge. net/.

${ }^{5}$ GNU Octave is a high-level language mostly compatible with Matlab. See http://www.octave.org/.
} 

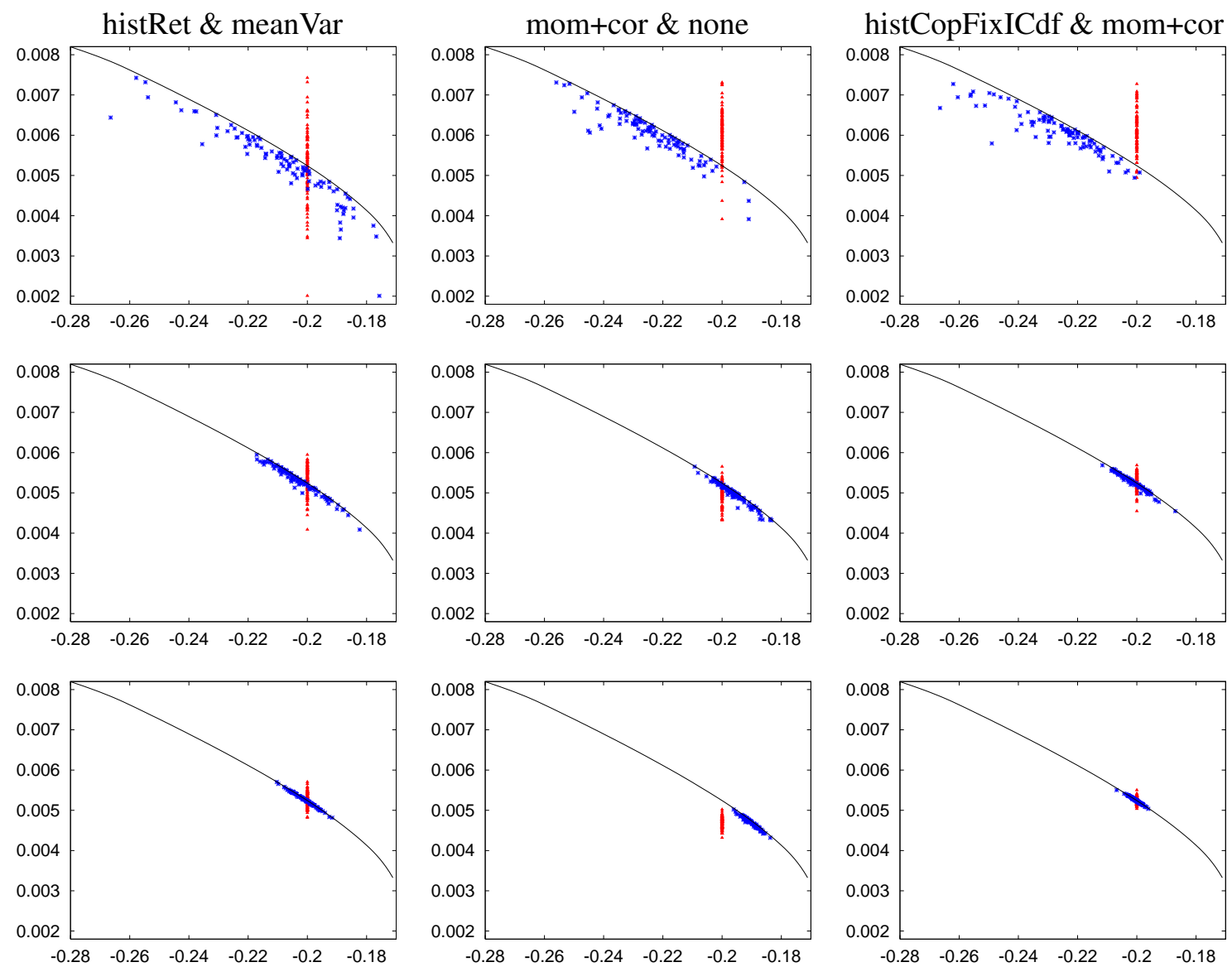

Figure 4: In-sample and Out-of-sample properties of three selected scenario generation methods, on trees with 50, 250, and 1000 scenarios. On the $x$-axis is CVaR, on the $y$-axis the objective function values. The in-sample values are scattered along a vertical line "CVaR $=-0.2$ ", caused by the constraint on CVaR. The rest of the points represent the out-of-sample values and the line represents the "true" CVaR-efficient frontier. Note that the in-sample values can be above the efficient frontier, since they do not represent the true (out-of-sample) values.

- Matching the moments and correlations of the historical returns without any direct use of the data leads to one of the most stable methods, but can introduce a bias to the results. This is due to the fact that starting without any particular structure basically implies the elliptical structure of the normal distribution. When the data has significantly different structure, this approach leads to a bias in the results.

In the last row of plots in Fig. 4, we can see that the moment-matching for 1000 scenarios led to smaller risk than required. However, in the case of CVaR constraint at -0.25 (instead of -0.2), the moment-matching resulted in portfolio with a higher-than-required risk. This illustrates that the bias caused by moment-matching is unpredictable, including the sign of the bias.

- Fixing the margins of the copulas to a fixed discretization improved the stability in most of the tests (and did not make it worse in the rest). As it did not introduce/increase the bias of the results as well, we conclude that the fixing of margins is a useful technique.

- Transforming the margins via the normal distribution (methods histCopNorm and histCop- 
FixNorm) may in some cases decrease stability or introduce a bias to the results. This is, again, not surprising as this method relies only on the four moments to set the margins, discarding thus the additional information from the empirical CDF. In addition, the cubic transformation is not guaranteed to be monotonous, so it can distort the copula, see Section 1.1.

- As expected, controlling the first four moments and correlations provides more stable results than controlling only the moments, which in turn is more stable than controlling only means and variances. ${ }^{6}$

\section{Conclusions}

In this article we have shown how to separate marginal distributions from the multivariate structurethe copula - when generating scenarios. This way we can combine different approaches which, separately, may be good for only one of these factors. We show that in some situations the combined approach retains both margins and copula, while in others we end up with approximations. By this separation of margins and copula it is for example possible to sample from the underlying distribution to obtain an approximation of the structure, while not having to rely on the same sample for margins. The margins can then be set up with other methods that are better suited to handle them, but which are possibly even unable to handle multivariate structure. Our example from portfolio management indicates that such an approach is indeed a good idea.

\section{Acknowledgements}

We would like to thank Pavel Popela and Pavla Zemánková from Brno University of Technology, Brno, Czechia, for their help with the initial draft of the paper. The authors have been supported under grant no. 156315/530 under "Strategisk Høgskoleprogram" from The Research Council of Norway. Furthermore, Michal Kaut has been supported by the project no. 103/05/0292 from the Grant Agency of the Czech Republic.

\section{References}

C. J. Adcock. Asset pricing and portfolio selection based on the multivariate skew-student distribution. Discussion Papers Series 2003-02, The Department of Economics, The University of Sheffield, 2003.

Adelchi Azzalini and Antonella Capitanio. Distributions generated by perturbation of symmetry with emphasis on a multivariate skew t-distribution. Journal of the Royal Statistical Society: Series B (Statistical Methodology), 65:367-389, 2003.

\footnotetext{
${ }^{6}$ There was, however, a strange effect in the case of 50 scenarios with the main data set. There, the trees generated with the moment-matching post-process introduced a bias so the out-of-sample risk was consistently bigger than the in-sample one. When only means and variances were corrected, the bias disappeared, though the results were significantly less stable. This effect has not been observed on the MSCI dataset or on trees with 250 or 1000 scenarios.

At the moment, we do not know what causes this behaviour, but it could be connected to the way we compute the (higher) moments, using population-based rather than sample-based formulas.
} 
Luc Bauwens and Sébastien Laurent. A new class of multivariate skew densities, with application to GARCH models. Computing in Economics and Finance 2002 5, Society for Computational Economics, 2002. available at http://ideas.repec. org/p/sce/scecf2/5.html.

Güzin Bayraksan and David P. Morton. Assessing solution quality in stochastic programs. Stochastic Programming E-Print Series, http://www . speps . info, 2005.

Eric Bouyé, Valdo Durrleman, Ashkan Nikeghbali, Gaël Riboulet, and Thierry Roncalli. Copulas for finance: A reading guide and some applications. working paper, Crédit Lyonnais, Paris, 2000.

Anukal Chiralaksanakul and David P. Morton. Assessing policy quality in multi-stage stochastic programs. Stochastic Programming E-Print Series, http://www. speps . info, 2004.

Robert T. Clemen and Terence Reilly. Correlations and copulas for decision and risk analysis. Management Science, 45(2):208-224, February 1999.

S. Demarta and A. J. McNeil. The $t$ copula and related copulas. International Statistical Review, 73 (1):111-129, 2005.

Victor DeMiguel and Nishant Mishra. A multistage stochastic programming approach to network revenue management. Lbs working paper, London Business School, 2006.

Jitka Dupačová, Giorgio Consigli, and Stein W. Wallace. Scenarios for multistage stochastic programs. Ann. Oper Res., 100:25-53, 2000. ISSN 0254-5330.

Jitka Dupačová, Nicole Gröwe-Kuska, and Werner Römisch. Scenario reduction in stochastic programming. Mathematical Programming, 95(3):493-511, 2003.

. ment Science, 47(2):295-307, 2001. 
Kjetil Høyland, Michal Kaut, and Stein W. Wallace. A heuristic for moment-matching scenario generation. Computational Optimization and Applications, 24(2-3):169-185, 2003. ISSN 0926-6003.

Ling Hu. Dependence patterns across financial markets: A mixed copula approach. Applied Financial Economics, 16(10):717-729, 2006.

Farshid Jamshidian and Yu Zhu. Scenario simulation: Theory and methodology. Finance and Stochastics, 1(1):43-67, 1996.

Eric Jondeau and Georg Michael Rockinger. Conditional volatility, skewness and kurtosis: Existence and persistence. Working Paper 77, Banque de France, Direction Generale des Etudes, 2000. Also appeared as HEC (Ecole des Hautes Etudes Commerciales) Department of Finance Working Paper No. $710 / 2000$.

M. C. Jones. Multivariate $t$ and beta distributions associated with the multivariate $f$ distribution. Metrika, 54:215-31, 2001.

Michal Kaut and Stein W. Wallace. Evaluation of scenario-generation methods for stochastic programming. Stochastic Programming E-Print Series, http: //www. speps . info, May 2003.

Michal Kaut, Stein W. Wallace, Hercules Vladimirou, and Stavros Zenios. Stability analysis of a portfolio management model based on the conditional value-at-risk measure. Feb 2003.

J. T. Linderoth, A. Shapiro, and S. J. Wright. The empirical behavior of sampling methods for stochastic programming. Optimization Online, http://www.optimization-online.org, 2002.

François Longin and Bruno Solnik. Extreme correlation of international equity markets. The Journal of Finance, 56(2):649-676, 2001.

Roger B. Nelsen. An Introduction to Copulas. Springer-Verlag, New York, 1998.

Andrew Patton. Skewness, asymmetric dependence, and portfolios. In PhD Thesis, chapter 3. Department of Economics, University of California, San Diego, 2002.

Andrew J. Patton. On the out-of-sample importance of skewness and asymmetric dependence for asset allocation. Journal of Financial Econometrics, 2(1):130-168, 2004.

G. C. Pflug. Scenario tree generation for multiperiod financial optimization by optimal discretization. Mathematical Programming, 89(2):251-271, 2001.

R. T. Rockafellar and S. Uryasev. Optimization of conditional value-at-risk. The Journal of Risk, 2 (3):21-41, 2000.

Claudio Romano. Calibrating and simulating functions: An application to the italian stock market. working paper 12, Centro Interdipartimale sul Diritto e l'Economia dei Mercati, 2002.

W. Römisch and H. Heitsch. Scenario reduction algorithms in stochastic programming. Computational Optimization and Applications, 24(2-3):187-206, 2003.

Joshua V. Rosenberg. Non-parametric pricing of multivariate contingent claims. The Journal of Derivatives, 10(3):9-26, 2003. 
A. Sklar. Fonctions de répartition à n dimensions et leurs marges. Publications de l'Institut de Statistique de l'Université de Paris, 8:229-231, 1959.

A. Sklar. Random variables, distribution functions, and copulas - a personal look backward and forward. In L. Rüschendorff, B. Schweizer, and M. Taylor, editors, Distributions with Fixed Marginals and Related Topics, pages 1-14. Institute of Mathematical Statistics, Hayward, CA, 1996.

6 Nikolas Topaloglou, Hercules Vladimirou, and Stavros A. Zenios. CVaR models with selective hedging for international asset allocation. Journal of Banking and Finance, 26(7):1535-1561, 2002.

8 S. Uryasev. Conditional value-at-risk: Optimization algorithms and applications. Financial Engineering News, (14):1-5, 2000. URL http: // fenews.com/.

10 S.W. Wallace and W.T. Ziemba, editors. Applications of Stochastic Programming. MPS-SIAM Series on Optimization, Philadelphia, 2005. 